\title{
Effects of Tai Chi Training on Antioxidant Capacity in Pre- and Postmenopausal Women
}

\author{
Attakorn Palasuwan, ${ }^{1,2}$ Daroonwan Suksom, ${ }^{3}$ Irène Margaritis, ${ }^{4}$ \\ Suphan Soogarun, ${ }^{2}$ and Anne-Sophie Rousseau ${ }^{1}$ \\ ${ }^{1}$ Faculté des Sciences du Sport, Université de Nice Sophia-Antipolis, 261 Route de Grenoble, 06205 Nice, France \\ ${ }^{2}$ Faculty of Allied Health Sciences, Chulalongkorn University, Bangkok 10330, Thailand \\ ${ }^{3}$ Faculty of Sports Science, Chulalongkorn University, Bangkok 10330, Thailand \\ ${ }^{4}$ French Agency for Food, Environmental and Occupational Health Safety, Maisons-Alfort, France \\ Correspondence should be addressed to Anne-Sophie Rousseau, asrousse@unice.fr
}

Received 8 August 2010; Revised 3 January 2011; Accepted 25 January 2011

Academic Editor: Ben Hurley

Copyright ( $\odot 2011$ Attakorn Palasuwan et al. This is an open access article distributed under the Creative Commons Attribution License, which permits unrestricted use, distribution, and reproduction in any medium, provided the original work is properly cited.

The risk of oxidative stress-related metabolic diseases increases with menopause and physical inactivity. We hypothesized that an 8-week Tai Chi (TC) training program (2 sessions in class; 2 sessions at home; 1-1:15/session) would improve antioxidant capacity and reduce cardiovascular risks in both pre- $(n=8)$ and postmenopausal $(n=7)$ sedentary women. Selected measures of physical fitness and blood parameters were analyzed before and after the program. Besides the well-known effects of TC on balance, flexibility, and maximum leg extensor strength, TC (1) increased erythrocyte glutathione peroxidase activity—an aerobic training-responsive antioxidant enzyme-and plasma total antioxidant status and (2) decreased plasma total homocysteine, a cardiovascular risk marker. In addition to being a low-velocity, low-impact, and relatively safe, TC is a suitable physical activity design for pre- and postmenopausal women to increase antioxidant defenses. Investigating breathing effects during TC movements would be an interesting area for further research in diseases prevention.

\section{Introduction}

The risk of oxidative stress-related metabolic diseases increases with menopause and physical inactivity $[1,2]$. The decline in exercise capability with aging contributes to physiological limitations, such as declines in muscle strength, flexibility, balance, and cardiovascular function [3]. Regular strength training can counteract, at least partly, this aging effect, improving functional ability and reducing the risk of fall [4]. However, it has little effect on cardiovascular risk [5] and antioxidant defense markers, which are elicited mostly by metabolic changes, induced by aerobic physical activity $[6$, 7]. Some clinical markers, such as oxidative stress markers, are associated with lung capacity that is inversely related to the increasing risk of metabolic diseases during menopause [8]. With aging exercise tasks are performed at a higher percentage of maximal aerobic capacity $\left(\mathrm{V}_{2} \mathrm{max}_{2}\right)$, shifting substrate use from lipid-derived energy to carbohydrate.
During menopause, the decreasing levels of estrogens reinforce the deterioration of both lipid metabolism and antioxidant status that is observed when the level of physical activity is chronically low $[9,10]$. Although physical activity has many benefits on health, some exercises are not suitable and not recommended for older women. The combination of inappropriate training loads (high intensity, high impact, low recovery time, etc.) and aging can lead to a failure of endogenous antioxidants to adapt to acute or chronic oxidative stress exposure $[11,12]$.

Interestingly, Tai Chi, an ancient Chinese mind-body exercise, is a martial art form combining breathing with rotational and multisegmental postures that flow smoothly from one side to the other side, through slow and gentle movements [13]. It is a weight-bearing exercise that strengthens muscle and which corresponds to an aerobic exercise of moderate intensity [14]. Tai Chi training has been shown to increase muscular strength, functional mobility, flexibility 
and balance, to improve sleep quality and duration and psychological well-being and to enhance microcirculatory function $[13,15,16]$. However, its possible effects on the adaptive antioxidant system have been largely ignored. Yogic "pranayama" breathing, an Indian mind-body exercise, has been shown to improve endogenous antioxidant capacity and to lower oxidative stress markers [17]. Breathing synchronization with cardiovascular rhythms may enhance cardiorespiratory performances as previously suggested [18]. Recently, we have shown in a cross-sectional study that preand postmenopausal women, who were regularly practicing Tai Chi, had a higher erythrocyte glutathione peroxidase (GPx) activity compared to their sedentary counterparts [19]. To confirm a potential antioxidant effect of Tai Chi training in women, we have conducted an interventional study. Eight weeks of Tai Chi training-combining classical Tai Chi postures with slow and deep breathing ( 6 breaths per minute)-were expected to induce not only functional, but also antioxidant effects. It was hypothesized that menopausal women would benefit from these adaptations. Particularly, we hypothesized that Tai Chi training would improve antioxidant capacity and reduce plasma lipid peroxidation as well as cardiovascular risk markers concentrations in preand postmenopausal women.

\section{Methods}

2.1. Subjects and Design. Eight healthy premenopausal (preM; $39 \pm 6$ yrs old) and 7 postmenopausal (post-M; $54 \pm$ 3 yrs old) women volunteered to participate in an 8-week Tai Chi (TC) training program (characteristics in Table 1). All participants were community-dwelling sedentary women who worked for the same organization in Bangkok. All participants completed inclusion and lifestyle questionnaires. Excluded subjects from this study were smokers, regular alcohol consumers, antioxidant supplement users, women treated with hormone replacement therapy, and women with renal, cardiovascular or hepatic disease, diabetes, asthma, or cancer. One participant did not attend the TC class regularly, and her data were excluded from statistical analysis. Given the condition of inclusion, sample sizes were smaller than expected, but groups were homogeneous according to their lifestyle. Participants were informed of the nature of the experiment before giving their formal consent. The protocol was approved by the Ethics Review Committee for Research Involving Human Subjects, Health Science Group of Faculties, Colleges and Institutes, Chulalongkorn University, Thailand (Approval no. 087/2008), constituted in accordance with the International Conference on HarmonizationGood Clinical Practice (ICH-GCP).

2.2. Experimental Design. The 8-week TC training protocol consisted of two training sessions per week with 75 min per session. In addition, all participants were given a video CD demonstrating Tai Chi postures and were asked to practice twice a week at home, with $60 \mathrm{~min}$ per session. Eligible participants were required to attend classes for at least $80 \%$ of the training program.
The class protocol included $5 \mathrm{~min}$ of check-in, $10 \mathrm{~min}$ of warm-up exercise (stretching), and $60 \mathrm{~min}$ of Tai Chi (comprising $45 \mathrm{~min}$ of 18-posture Tai Chi and $15 \mathrm{~min}$ of Tai Chi Fan style). Classical Tai Chi postures are characterized by slow rotational movements performed in semisquat postures with varying degrees of arm movement. Not only lowerbody balance by leg and knee extension, but also upperbody flexibility by arm contractions, was exercised. The TC training program was a modified 18-movement Yang style with three versions. Each motion was performed slowly coupling breathing to music, and usually took 1$1.5 \mathrm{~min}$ per motion or $18-25 \mathrm{~min}$ per completed set. The TC practitioners imitated the movements of the experienced TC instructor at the same speed. The major requirement of this modified 18-posture was that the TC practitioners had to concentrate and carefully control the slow and deep breathing with a frequency of 6 breaths per minute (or 10 seconds per breath). To ensure the breathing rhythm and control the time course, the movements were performed to traditional Chinese music that has sound signals for inhalation. Five to ten movements were taught each week, so a complete 18-postured set was practiced for 2 weeks, and three versions of the 18-postured set were completed in 6 weeks. After performing conventional TC in each session, all participants were also taught TC fan style, which combined TC training and a fan dance with traditional Chinese music. During the class, the instructor monitored and corrected the posture of each subject. To ensure that the participants were practicing at home, participants had to complete a training $\log$ in which they had to report details about their home practice.

Anthropometric data, dietary intakes, blood draws, physical activity, and selected measures of physical fitness blood were assessed at the beginning and after 8 weeks of TC training. All participants were asked to maintain their usual dietary behavior and activity habits.

2.3. Anthropometric Data. Body weight was measured with a calibrated electrical balance (Tanita, Inner Scan BC533 model, Japan). Resting blood pressure and pulse were measured with a portable blood pressure monitor (Omron Hypertension HEM 780, Japan). The percentage of body fat was assessed using the standard Lange skinfold method (3 positions: triceps, suprailiac and thigh). Skinfolds readings were taken from triceps, suprailiac and thigh sites for each subject. A Lange skinfold caliper was used throughout and a single well-trained technician made all readings. Lange skinfolds were measured three times and the average of the three was recorded. Percentages of body fat were calculated by Jackson's formula [20].

\subsection{Diet and Activity Records}

2.4.1. Dietary Intakes. Before each venipuncture, each subject completed, at home, a 4-day food diary (including a weekend day). A standardized information session was organised to give each subject instructions on how to record their daily food intake and activities. Food quantities were 
TABLE 1: Characteristics of pre- and post-menopausal women before and after the Tai Chi training period.

\begin{tabular}{|c|c|c|c|c|}
\hline & \multicolumn{2}{|c|}{ Pre-M women } & \multicolumn{2}{|c|}{ Post-M women } \\
\hline & Pre-training & Post-training & Pre-training & Post-training \\
\hline Age (yrs) & $39 \pm 6$ & $39 \pm 6$ & $54 \pm 3^{\#}$ & $54 \pm 3^{\#}$ \\
\hline Height $(\mathrm{cm})$ & $160 \pm 4$ & $160 \pm 4$ & $155 \pm 5$ & $155 \pm 5$ \\
\hline Body weight (kg) & $56 \pm 10$ & $56 \pm 10$ & $63 \pm 9$ & $62 \pm 9$ \\
\hline BMI $\left(\mathrm{kg} \cdot \mathrm{m}^{-2}\right)$ & $21.9 \pm 4.0$ & $21.9 \pm 4$ & $26.2 \pm 3.2^{\#}$ & $25.8 \pm 3.0^{\#}$ \\
\hline Body fat (\%) & $31.2 \pm 6.4$ & $30.2 \pm 6.5^{*}$ & $38.2 \pm 4.2$ & $33.8 \pm 5.1^{*}$ \\
\hline SBP (mmHg) & $113 \pm 13$ & $114 \pm 15$ & $142 \pm 18^{\#}$ & $136 \pm 22^{\#}$ \\
\hline DBP (mmHg) & $72 \pm 9$ & $79 \pm 18$ & $92 \pm 14$ & $79 \pm 9$ \\
\hline Resting HR (beats $\cdot \min ^{-1}$ ) & $84 \pm 17$ & $88 \pm 15$ & $78 \pm 10$ & $78 \pm 13$ \\
\hline Balance (sec) & $19.3 \pm 2.1$ & $15.6 \pm 1.3^{*}$ & $22.2 \pm 1.8^{\#}$ & $17.9 \pm 2.5^{* \#}$ \\
\hline Flexibility $(\mathrm{cm})$ & $10.4 \pm 6.0$ & $12.6 \pm 5.4^{*}$ & $8.4 \pm 4.8$ & $11.4 \pm 5.3^{*}$ \\
\hline 1-RM load leg curl (kg) & $27.9 \pm 3.9$ & $32.9 \pm 3.9$ & $26.4 \pm 4.8$ & $31.4 \pm 4.8$ \\
\hline 1-RM load leg extension (kg) & $42.9 \pm 4.9$ & $48.6 \pm 8.0^{*}$ & $45.0 \pm 5.8$ & $52.1 \pm 7.0^{*}$ \\
\hline 1-RM load biceps curl (kg) & $7.1 \pm 3.7$ & $9.3 \pm 4.5$ & $5.7 \pm 4.0$ & $7.1 \pm 3.4$ \\
\hline 1-RM load biceps extension (kg) & $12.9 \pm 0.9$ & $13.6 \pm 1.3$ & $12.1 \pm 2.2$ & $13.6 \pm 2.0$ \\
\hline $\mathrm{VO}_{2 \max }\left(\mathrm{mL} \cdot \mathrm{kg}^{-1} \cdot \mathrm{min}^{-1}\right)$ & $25.9 \pm 3.4$ & $25.7 \pm 4.4$ & $22.1 \pm 2.2^{\#}$ & $21.1 \pm 2.3^{\#}$ \\
\hline
\end{tabular}

Values are expressed as mean \pm standard deviation; ${ }^{*} P<.005$ : different from Pre-training in Pre-M and Post-M; ${ }^{*} P<.005:$ different from Pre-M in Pre-training and Post-training; Pre-M: premenopausal women group; Post-M: postmenopausal women group; BMI: body mass index; SBP: systolic blood pressure; DBP: diastolic blood pressure; HR: heart rate.

estimated using standard measures for a cup, tablespoon, and teaspoon (validated by the Institute of Nutrition, Mahidol University) and specifying the number of units/code corresponding to the size of the food portion by comparison with photos from an adapted portion book. Nutrient intakes were calculated using INMUCAL nutrient software, version 2007, which uses the Thai dietary database of the Institute of Nutrition, Mahidol University, and the Nutrition Division, Department of Health, Ministry of Public Health of Thailand (version 2003).

2.4.2. Physical Activity Record. In parallel to dietary records, subjects completed a 4-day activity records diary. The 4-day physical activities diary was divided according to categories of physical activity [21] ((1) sleep position; (2) sitting position activities; (3) standing position including small movements; (4) walking, gardening, etc.; (5) Tai Chi training). The compendium of physical activities [22] was used to estimate the energy cost of physical activity expressed as metabolic equivalents (METs). The appropriate MET values, based on the subject's report of the type and intensity of activity, were assigned. Daily energy expenditure (EE) was calculated by multiplying the amount of time spent in each activity and the corresponding MET, using a computer program specifically designed for this task. Activities not found in the database were carefully evaluated to determine the best-suited corresponding activity. MET values for TC training was set at $3 \mathrm{MET}$ as previously measured by Chao et al. [23] and confirmed [24].

2.5. Physical Fitness Assessments. For all subjects and conditions, on the morning, a balance test was performed first and a muscle strength test second. In the afternoon, a maximal aerobic capacity test $\left(\dot{\mathrm{V}}_{2}\right.$ max $)$ was performed. The next day, on the morning, subjects came back in the lab to perform a flexibility test. Except for $\dot{\mathrm{V}}_{2}$ max determination, tests were done in triplicate (every 30 minutes) and began with a warm up of 3-5 minutes. The best score was recorded.

2.5.1. Balance Test. Balance was tested by a timed up and go test. The participant sat on a chair and waited for the start signal. At the signal, the participant had to rapidly stand up, to walk quickly across two cones on the way, and to return to a seated position in the same chair. When the participant came back to the starting point, the total time was recorded. The participant was instructed to perform the test as quickly as possible [25].

2.5.2. Flexibility Test. Flexibility was measured with the sit and reach test [26]. Subjects sat on the floor with legs extended, reached forward with their hands, one placed on top of the other, and held the terminal position for at least 2 seconds. The score is recorded to the nearest centimeter as the distance reached by the hand.

2.5.3. Muscle Strength Tests. Muscle strength was measured by dynamic testing [27] on standard weight machines (Marathon, Thailand). The one-repetition maximum (1RM) strength was evaluated in four different exercises: the biceps curl, triceps extension, leg extension (quadriceps), and leg curl (hamstring).

2.5.4. Maximal Aerobic Capacity. Subjects performed a continuous incremental walking ( $3 \mathrm{~min}$ up to $8 \mathrm{~km} \cdot \mathrm{h}^{-1}$ ) and running test $\left(1 \mathrm{~km} \cdot \mathrm{h}^{-1}\right.$ increment every 2 min to exhaustion) on a motorized treadmill $(\mathrm{h} / \mathrm{p} /$ cosmos mercury, Germany) 
with a $1 \%$ slope. Ventilation and gas exchanges were measured using a breath-by-breath gas analysis system (Cortex Metamax 3B, Germany). Heart rate was recorded using a telemetric system (Polar Electro S810, Finland). The $\mathrm{VO}_{2}$ peak was determined from the observation of at least two of the following criteria: $\mathrm{VO}_{2}$ was increased less than $2 \mathrm{~mL} \cdot \mathrm{min}^{-1} \cdot \mathrm{kg}^{-1}$ after 2 minutes, HR exceeded its agepredicted maximum, and the respiratory exchange ratio (RER) exceeded 1.15.

2.6. Blood Samples and Assays. Blood samples were collected from a forearm vein in $\mathrm{K}_{3}$ EDTA tubes $(3 \mathrm{~mL})$ and $\mathrm{Li}$ heparin tubes $(6 \mathrm{~mL})$ (Vacuette, Greiner Bio-One, Germany) between 7.00 and 9.30 AM after an overnight fast. Heparinized plasma was removed by centrifugation at $3000 \times \mathrm{g}$ at $4^{\circ} \mathrm{C}$ for $15 \mathrm{~min}$ within an hour after blood collection for total antioxidant status, lipid peroxidation, and total homocysteine concentration ( $\mathrm{tHcy}$ ) analyses. Heparinized whole blood $(0.05 \mathrm{~mL})$ was diluted with $1 \mathrm{~mL}$ of diluting agent for glutathione peroxidase (GPx) (Randox laboratories, UK) and kept at $-80^{\circ} \mathrm{C}$ until GPx analysis. The packed red blood cells (RBCs) were washed three times with two volumes of isotonic saline solution at $\mathrm{pH}$ 7.0. The washed RBCs were hemolyzed by suspension in double distilled water, centrifuged at $3000 \times \mathrm{g}$ at $4^{\circ} \mathrm{C}$ for $15 \mathrm{~min}$, and then kept at $-80^{\circ} \mathrm{C}$ for erythrocyte GPx and superoxide dismutase (SOD) activity analysis. Each assay of antioxidant was performed on the same day to eliminate variation in assay conditions. The samples were stored in Eppendorf tubes at $-80^{\circ} \mathrm{C}$ and thawed only once before analysis to eliminate a freeze-thaw effect. All assays for each marker were performed with the same conditions (same set of reagent kit, same control, same automate) on the same day, to avoid interassay variations. The automate system was set to randomly repeat the control during the batch to avoid run-to-run variations. The withinrun coefficient of variation was less than $2.5 \%$ for the biochemical markers measured.

2.7. Biological Assays. Total antioxidant status (TAS) concentration was measured by the radical cation decolorization assay [28] using spectrophotometer (Shidmadzu UV-1601, Japan) at $734 \mathrm{~nm}$. Lipid peroxidation (TBARS) was assayed by measuring the formation of malondialdehyde [29] and measured by spectrophotometer at $530 \mathrm{~nm}$ (Shidmadzu UV-1601, Japan). Superoxide dismutase and GPx activities were detected by enzyme kinetic-colorimetric assay (Randox Laboratories, UK) using biochemical automatic analyzer (Vitalab Selectra XL, Flexor Company, The Netherlands). Plasma total homocysteine (tHcy) concentrations were analyzed by Fluorescence Polarization Immunoassay using the AxSYM automatic system (Abbott Laboratories, USA).

2.8. Statistical Analysis. Comparisons between groups (preand post-M) and between pre- and post-TC training intervention and investigations of the interaction effect between the TC training intervention and the menopausal status were made by a one-way analysis of variance (ANOVA) with repeated measures. When appropriate, the protected Fisher's

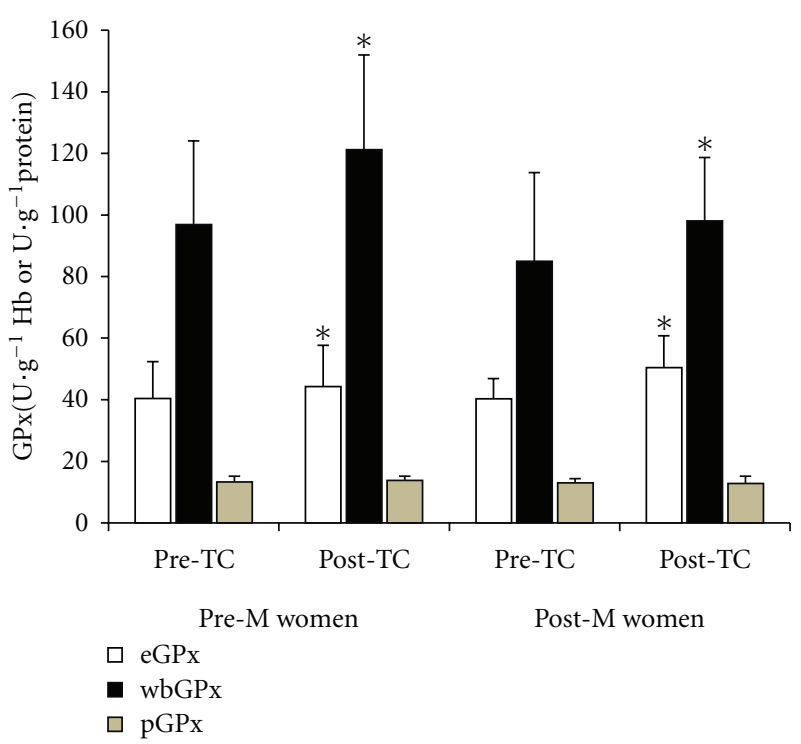

Figure 1: Effect of Tai Chi training on erythrocyte (eGPx, unit $\cdot \mathrm{g}^{-1} \mathrm{Hb}$ ), whole blood (wbGPx, unit $\cdot \mathrm{g}^{-1} \mathrm{Hb}$ ), and plasma ( $\mathrm{pGPx}$, unit. $\mathrm{g}^{-1}$ total protein) glutathione peroxidase activities in pre-menopausal (Pre-M) and post-menopausal menopausal (Post$\mathrm{M})$ women. ${ }^{*} P<.001$, different from Pre-training.

Least Significant Difference (LSD) test was performed. When parameters did not follow a normal distribution, we treated them as nonparametric variables using Mann \& Whitney or Wilcoxon signed ranks tests. Statistical analyses were made using SPSS (version 15) software. Significance was established at $P<.05$. Values are reported as mean \pm standard deviation (SD).

\section{Results}

3.1. Baseline Differences between Pre- and Post-M Women. At baseline, post-M women had significantly lower balance and $\dot{\mathrm{V}}_{2}$ max compared with pre-M women (Table 1 ). No difference was found between pre-M and post-M women regarding other selected physical fitness parameters (Table 1). Post-M women had significantly higher systolic blood pressure (Table 1). Daily energy expenditure and daily energy intake were not significantly different in pre-M compared to post-M women (Table 2). Post-M women had lower animalderived proteins intake compared to pre-M women but the daily intake of macronutrients and of selected micronutrients was not significantly different between groups (Table 2). LDL cholesterol tended to be higher $(P=.08)$ in post-M women compared to pre-M women. No difference between groups was shown in antioxidant status and in other selected biochemical parameters (Table 3, Figure 1).

3.2. Tai Chi Training Effect. Compared to pretraining data, balance, flexibility, and leg extension strength, were significantly improved in both pre-M and post-M women after the TC training protocol (Table 1). No significant change was observed on systolic and diastolic blood pressure, 
TABle 2: Daily energy expenditure and daily nutrient intake of pre- and post-menopausal women before and after the Tai Chi training period.

\begin{tabular}{|c|c|c|c|c|}
\hline & \multicolumn{2}{|c|}{ Pre-training } & \multicolumn{2}{|c|}{ Post-training } \\
\hline & Pre M & Post M & Pre M & Post M \\
\hline $\mathrm{EE}\left(\mathrm{kCal} \cdot \mathrm{d}^{-1}\right)$ & $1770 \pm 206$ & $1821 \pm 185$ & $1868 \pm 285^{*}$ & $2024 \pm 318^{*}$ \\
\hline $\mathrm{EI}\left(\mathrm{kCal} \cdot \mathrm{d}^{-1}\right)$ & $1579 \pm 364$ & $1311 \pm 260$ & $1576 \pm 566$ & $1617 \pm 140$ \\
\hline Carbohydrate $\left(\mathrm{g} \cdot \mathrm{d}^{-1}\right)$ & $216 \pm 38$ & $190 \pm 36$ & $208 \pm 101$ & $209 \pm 26$ \\
\hline$\% \mathrm{EI}$ & $55.3 \pm 5.4$ & $57.9 \pm 4.8$ & $51.5 \pm 7.2^{*}$ & $51.6 \pm 5.2^{*}$ \\
\hline Fat $\left(g \cdot d^{-1}\right)$ & $50 \pm 19$ & $40 \pm 11$ & $55 \pm 19^{*}$ & $58 \pm 10^{*}$ \\
\hline$\% \mathrm{EI}$ & $27.5 \pm 4.5$ & $27.1 \pm 4.0$ & $31.6 \pm 6.2^{*}$ & $32.3 \pm 4.1^{*}$ \\
\hline $\operatorname{Protein}\left(\mathrm{g} \cdot \mathrm{d}^{-1} \cdot \mathrm{kg}^{-1}\right)$ & $1.246 \pm 0.318$ & $0.826 \pm 0.400$ & $1.141 \pm 0.216$ & $1.068 \pm 0.205$ \\
\hline$\% \mathrm{EI}$ & $17.1 \pm 2.0$ & $15.0 \pm 3.3$ & $16.9 \pm 3.6$ & $16.0 \pm 1.6$ \\
\hline Animal protein $\left(\mathrm{g} \cdot \mathrm{d}^{-1} \cdot \mathrm{kg}^{-1}\right)$ & $0.690 \pm 0.172$ & $0.405 \pm 0.288^{\#}$ & $0.597 \pm 0.152$ & $0.562 \pm 0.156^{\#}$ \\
\hline Vegetal protein $\left(\mathrm{g} \cdot \mathrm{d}^{-1} \cdot \mathrm{kg}^{-1}\right)$ & $0.322 \pm 0.137$ & $0.282 \pm 0.195$ & $0.310 \pm 0.138$ & $0.306 \pm 0.129$ \\
\hline$\beta$-Carotene $\left(\mu \mathrm{g} \cdot \mathrm{d}^{-1}\right)$ & $1150 \pm 834$ & $867 \pm 765$ & $803 \pm 572$ & $887 \pm 585$ \\
\hline Vitamin $C\left(\mathrm{mg} \cdot \mathrm{d}^{-1}\right)$ & $175 \pm 156$ & $107 \pm 81$ & $96 \pm 155$ & $145 \pm 199$ \\
\hline Vitamin $\mathrm{E}\left(\mathrm{mg} \cdot \mathrm{d}^{-1}\right)$ & $6.91 \pm 11.80$ & $9.00 \pm 15.93$ & $10.06 \pm 13.91$ & $6.58 \pm 11.87$ \\
\hline
\end{tabular}

Pre M: pre-menopausal women group; Post M: post-menopausal women group; EE: energy expenditure; EI: energy intake. Values are expressed as mean \pm standard deviation; ${ }^{*} P<.05$, different from pre-training; ${ }^{\#} P<.05$, different from Pre M.

TABLE 3: Hematological and biochemical parameters of pre- and post-menopausal women before and after the Tai Chi training period.

\begin{tabular}{|c|c|c|c|c|}
\hline & \multicolumn{2}{|c|}{ Pre-M women } & \multicolumn{2}{|c|}{ Post-M women } \\
\hline & Pre-training & Post-training & Pre-training & Post-training \\
\hline Hemoglobin $\left(\mathrm{g} \cdot \mathrm{dL}^{-1}\right)$ & $13.06 \pm 0.94$ & $13.00 \pm 0.86$ & $13.76 \pm 0.56$ & $13.31 \pm 0.63$ \\
\hline Hematocrit (g\%) & $39.0 \pm 3.1$ & $38.4 \pm 2.7$ & $40.9 \pm 1.9$ & $40.1 \pm 2.3$ \\
\hline Leukocytes $\left(\times 10^{9} \mathrm{~L}^{-1}\right)$ & $5.93 \pm 1.34$ & $5.90 \pm 1.13$ & $5.93 \pm 1.06$ & $5.27 \pm 1.07$ \\
\hline Platelets $\left(\times 10^{9} \mathrm{~L}^{-1}\right)$ & $290 \pm 110$ & $295 \pm 125$ & $254 \pm 42$ & $227 \pm 46$ \\
\hline Uric acid $\left(\mathrm{mg} \cdot \mathrm{dL}^{-1}\right)$ & $3.19 \pm 0.59$ & $3.24 \pm 0.66$ & $4.09 \pm 1.41$ & $4.44 \pm 1.27$ \\
\hline Triglycerides $\left(\mathrm{mg} \cdot \mathrm{dL}^{-1}\right)$ & $57 \pm 18$ & $65 \pm 33$ & $99 \pm 50$ & $88 \pm 29$ \\
\hline Total cholesterol $\left(\mathrm{mg} \cdot \mathrm{dL}^{-1}\right)$ & $212 \pm 32$ & $203 \pm 30$ & $226 \pm 24$ & $217 \pm 33$ \\
\hline HDL cholesterol $\left(\mathrm{mg} \cdot \mathrm{dL}^{-1}\right)$ & $71 \pm 34$ & $72 \pm 35$ & $54 \pm 6$ & $56 \pm 7$ \\
\hline LDL cholesterol $\left(\mathrm{mg} \cdot \mathrm{dL}^{-1}\right)$ & $129 \pm 19$ & $118 \pm 19^{*}$ & $152 \pm 25$ & $143 \pm 33^{*}$ \\
\hline TAS $\left(\mathrm{mmol} \cdot \mathrm{L}^{-1} \mathrm{TE}\right)$ & $1.513 \pm 0.133$ & $1.697 \pm 0.122^{* *}$ & $1.573 \pm 0.087$ & $1.662 \pm 0.097^{* *}$ \\
\hline TBARS $(\mu \mathrm{M})$ & $2.44 \pm 2.07$ & $3.46 \pm 4.27$ & $2.12 \pm 1.21$ & $2.38 \pm 2.29$ \\
\hline eSOD (unit $\left.\cdot g^{-1} \mathrm{Hb}\right)$ & $1370 \pm 286$ & $1275 \pm 107$ & $1270 \pm 174$ & $1371 \pm 96$ \\
\hline $\mathrm{tHcy}\left(\mu \mathrm{mol} \cdot \mathrm{L}^{-1}\right)$ & $10.85 \pm 2.06$ & $8.87 \pm 1.88^{*}$ & $11.44 \pm 1.88$ & $9.51 \pm 1.81^{*}$ \\
\hline
\end{tabular}

Values are expressed as mean \pm standard deviation; Pre-M: premenopausal women group; Post-M: postmenopausal women group; TAS: total antioxidantstatus; TE: troloq equivalence; TBARS: plasma thiobarbituric acid reactive substance concentration; eSOD: erythrocyte superoxide dismutase activity; tHcy: plasma total homocysteine; ${ }^{*} P<.05$, different from Pre-training; ${ }^{*} P<.001$, different from Pre-training.

resting heart rate and $\dot{\mathrm{VO}}_{2 \text { max }}$ (Table 1). A slight but significantly higher total daily energy expenditure $(+3 \%$ in pre- $\mathrm{M}$ and $+6 \%$ in post- $\mathrm{M}$ women) was recorded after the TC training protocol (Table 2). Daily fat intake was recorded to be higher after the TC protocol (Table 2) but no significant difference was shown for other macronutrients and for total daily energy intake (Table 2). Moreover, the intake of antioxidant micronutrients remained unchanged (Table 2).

Compared to pre-training data, LDL cholesterol was lower $(P=.02 ; F=7.7)$ after the TC training protocol (Table 3). Total cholesterol, HDL cholesterol, triglycerides, uric acid and other routine blood parameters remained unchanged (Table 3). Concerning the antioxidant status, plasma TAS concentration $(P<.001 ; F=25.1)$ (Table 3$)$, erythrocyteGPx activity $(P=.001 ; F=19.0)$ and whole blood GPx activity $(P=.001 ; F=21.3)$ were significantly higher after the TC training protocol compared to pretraining data (Figure 1). However, erythrocyte SOD (Table 3 ) and plasma GPx activities (Figure 1) remained unchanged. Plasma tHcy concentration was significantly lower $(P<.001$; $F=44.6)$ after the TC training intervention compared to pre-training data (Table 3 ).

\subsection{Tai Chi Training and Menopausal Status Interaction} Effect. As shown by ANOVA tests, TC training effects were not different among groups (pre-M women versus post-M women). 


\section{Discussion}

The loss of the protective effect of estrogen in postmenopausal women is known to contribute to the increase in oxidative stress markers and to increase the risk of cardiovascular diseases [1]. This study investigated, whether a TC training protocol (1) would not only improve selected health-related fitness parameters but also the antioxidant capacityof preand post- $M$ women, and (2) would reduce oxidative damages and cardiovascular risks of post- $\mathrm{M}$ women.

In agreement with previous reports [16], our TC training program improved functional balance, muscle strength and flexibility of both pre- and post-M women. Plasma tHcy level, which is used as a risk marker of cardiovascular diseases, has been shown to increase after menopause [30]. Thus, we expected that TC training would reduce the level of this marker in post-M women. The TC program produced an $18 \%$ and $17 \%$ reduction in plasma tHcy level in pre$\mathrm{M}$ and post-M groups, respectively, but this effect did not depend on menopausal status. In addition, we have shown that our TC training program was able to improve erythrocyte and whole blood GPx activities in both pre$\mathrm{M}$ and post-M women. Cellular GPx plays a major role in the cellular defense against oxidative stress. The deficiency of cellular GPx in mice induced endothelial dysfunction and significant structural vascular and cardiac abnormalities [31]. Our previous reports showed that endurance exercise training improves erythrocyte GPx activity and that the elderly can also benefit from this adaptation $[6,12,32]$. Tai Chi exercises are performed at low to moderate intensity but seem to induce similar effects on this marker. We have paid very close attention to the breathing rate during TC movements ( 6 breathes/min or $10 \mathrm{sec} /$ breath). In comparison with a normal breathing frequency (15 breathes/min), this slower breathing frequency is synchronized to endogenous circulatory rhythms and improves cardiovascular and respiratory functions [17]. Respiration and physical activity can influence the autonomic control system, which in turn modify the rhythmic fluctuations in heart and blood pressure. This fluctuation may induce the activation of redoxsensitive transcription factors such as NF- $\kappa$ B and AP-1, which mediate the cellular mechanisms that initiate adaptive responses by changes in the GPx gene expression [33]. Some yogic rhythmic breathing training has been shown to improve endogenous antioxidant capacity [17] and to increase the expression of the glutathione S-transferase gene [34].

The relation between the increase in blood GPx activity and tHcy level is unclear but Hcy has been shown to decrease the ability of cells to detoxify hydrogen peroxide by impairing intracellular antioxidant enzymes, specifically GPx [35]. Moreover, the overexpression of cellular GPx can compensate for homocystemia effects $[36,37]$. Thus, it is possible that an increase in GPx activity with TC training directly or indirectly decreases the level of plasma tHcy.

The peroxyl radical scavenging capacity in plasma-also called plasma total antioxidant status (TAS) - was determined before and at the end of the training period. Although the difference between pre- and posttraining was very small
$(0.184 \mathrm{mM}(+12 \%)$ in pre-M women and $0.089 \mathrm{mM} \mathrm{TE}$ $(+6 \%)$ in post-M women $)$, the significance $(P<.001$; $F=25.1)$ was strong. Values did not seem to depend on plasma uric acid concentrations and evaluated dietary intakes, which remained unchanged. We used extremely controlled conditions (randomisation, standardisation, analysis performed on the same day, automatic analyzer, etc.) but the lack of control group remained a limit in the interpretation of our data changes. Thus, statistical power, particularly for TAS, was high despite the small number of subjects.

Although TAS and erythrocyte GPx activity were increased in our study, we failed to observe any significant change in the lipid peroxidation marker (TBARS), which nevertheless showed high interindividual variability. As already observed $[6,11,12,32]$, this marker may not be sensitive enough to detect variations in lipid peroxidation when it is measured in basal state (i.e., at rest), in a nonchallenging situation.

We failed to evidence significant interaction effect between menopause status and TC training. However, the increase in erythrocyte GPx showed in response to TC training, tended $(P=.09)$ to be of lower magnitude in pre-M compared to post-M women. Given the condition of inclusion, sample sizes were smaller than expected (15 subjects per groups for a statistical power of 80 percent). This suggests that post-M women would benefit more from this adaptation than pre-M women.

The clinical relevance of the improvement of erythrocyte GPx activity shown in our study is unknown. However, especially with aging, erythrocyte GPx activity may be a sensitive marker of the reinforcement of the endogenous antioxidant potential induced by regular physical activity training, as previously suggested in men [12]. The present study showed that it might be also the case for middle age and post- $\mathrm{M}$ women with Tai Chi training that is performed at a lower intensity and confirm our previous data on this marker obtained from a cross-sectional study [19].

\section{Conclusion}

The 8-week design TC training (2 sessions in class; 2 sessions at home, $1-1: 15 /$ session) is beneficial on functional balance, flexibility and muscular strength, not only for premenopausal women, but also for postmenopausal women. Besides the low-velocity, low-impact, and safety benefits, the main finding of this pilot study was that rotational movements of TC postures with slow and deep breathing increased erythrocyte GPx activity and reduced tHcy concentrations, in both pre- and postmenopausal women. Tai Chi would be a good physical activity design for aged women in order to increase their antioxidant protection and to prevent oxidative stress-related metabolic diseases. Further studies with larger groups are needed to confirm our presented results. Investigating breathing effects during TC movements would be an interesting area for further research in diseases prevention. 


\section{Acknowledgments}

The authors would like to thank Mr. Somsak Inpueak, Tai Chi instructor from Miracle Tai Chi Centre, for his instruction and Mr. Weerapat Yodkamolsad (Faculty of Sport Science, Chulalongkorn University) for his assistance in physical assessment and Dr. Isabelle Mothe-Satney (Inserm U907) for her critical reading. This work was partially supported by The Ratchadapisek Sompot Endowment Research Fund (for Royal Silver Jubilee of King Rama IX) of Chulalongkorn University. The authors thank all persons who participated in this study.

\section{References}

[1] J. E. Castelao and M. Gago-Dominguez, "Risk factors for cardiovascular disease in women: relationship to lipid peroxidation and oxidative stress," Medical Hypotheses, vol. 71, no. 1, pp. 39-44, 2008.

[2] U. Gaspard, "Hyperinsulinaemia, a key factor of the metabolic syndrome in postmenopausal women," Maturitas, vol. 62, no. 4, pp. 362-365, 2009.

[3] G. A. Heckman and R. S. McKelvie, "Cardiovascular aging and exercise in healthy older adults," Clinical Journal of Sport Medicine, vol. 18, no. 6, pp. 479-485, 2008.

[4] N. Beyer, L. Simonsen, J. Bülow et al., "Old women with a recent fall history show improved muscle strength and function sustained for six months after finishing training," Aging-Clinical and Experimental Research, vol. 19, no. 4, pp. 300-309, 2007.

[5] M. Y. Cortez-Cooper, M. M. Anton, A. E. DeVan, D. B. Neidre, J. N. Cook, and H. Tanaka, "The effects of strength training on central arterial compliance in middle-aged and older adults," European Journal of Cardiovascular Prevention and Rehabilitation, vol. 15, no. 2, pp. 149-155, 2008.

[6] F. Tessier, I. Margaritis, M. J. Richard, C. Moynot, and P. Marconnet, "Selenium and training effects on the glutathione system and aerobic performance," Medicine and Science in Sports and Exercise, vol. 27, no. 3, pp. 390-396, 1995.

[7] V. G. Coffey and J. A. Hawley, "The molecular bases of training adaptation," Sports Medicine, vol. 37, no. 9, pp. 737-763, 2007.

[8] F. G. Real, C. Svanes, E. R. Omenaas et al., "Lung function, respiratory symptoms, and the menopausal transition," Journal of Allergy and Clinical Immunology, vol. 121, no. 1, pp. 72-80, 2008.

[9] D. L. Enns and P. M. Tiidus, "The influence of estrogen on skeletal muscle: sex matters," Sports Medicine, vol. 40, no. 1, pp. 41-58, 2010.

[10] S. E. Campbell and M. A. Febbraio, "Effect of ovarian hormones on mitochondrial enzyme activity in the fat oxidation pathway of skeletal muscle," American Journal of PhysiologyEndocrinology and Metabolism, vol. 281, no. 4, pp. E803-E808, 2001.

[11] I. Margaritis and A. S. Rousseau, "Does physical exercise modify antioxidant requirements?" Nutrition Research Reviews, vol. 21, no. 1, pp. 3-12, 2008.

[12] A. S. Rousseau, I. Margaritis, J. Arnaud, H. Faure, and A. M. Roussel, "Physical activity alters antioxidant status in exercising elderly subjects," Journal of Nutritional Biochemistry, vol. 17, no. 7, pp. 463-470, 2006.

[13] C. Wang, J. P. Collet, and J. Lau, "The effect of Tai Chi on health outcomes in patients with chronic conditions: a systematic review," Archives of Internal Medicine, vol. 164, no. 5, pp. 493-501, 2004.

[14] C. Lan, S. Chen, and J. Lai, "The exercise intensity of Tai Chi Chuan," Medicine and Sport Science, vol. 52, pp. 12-19, 2008.

[15] G. Y. Yeh, C. Wang, P. M. Wayne, and R. S. Phillips, "The effect of Tai Chi exercise on blood pressure: a systematic review," Preventive Cardiology, vol. 11, no. 2, pp. 82-89, 2008.

[16] C. Lan, J. S. Lai, M. K. Wong, and M. L. Yu, "Cardiorespiratory function, flexibility, and body composition among geriatric Tai Chi Chuan practitioners," Archives of Physical Medicine and Rehabilitation, vol. 77, no. 6, pp. 612-616, 1996.

[17] S. Bhattacharya, U. S. Pandey, and N. S. Verma, "Improvement in oxidative status with yogic breathing in young healthy males," Indian Journal of Physiology and Pharmacology, vol. 46, no. 3, pp. 349-354, 2002.

[18] L. Bernardi, C. Porta, L. Spicuzza et al., "Slow breathing increases arterial baroreflex sensitivity in patients with chronic heart failure," Circulation, vol. 105, no. 2, pp. 143-145, 2002.

[19] A. Palasuwan, I. Margaritis, S. Sugaroon, and A. S. Rousseau, "Dietary intakes and antioxidant status in pre- and postmenopausal mind-body exercising women," Journal of Nutrition, Health \& Aging. In press.

[20] A. S. Jackson, M. L. Pollock, and A. Ward, "Generalized equations for predicting body density of women," Medicine and Science in Sports and Exercise, vol. 12, no. 3, pp. 175-182, 1980.

[21] M. Vermorel, P. Ritz, L. Tappy, and M. Laville, "Energie," in Apport Nutritionnels Conseillés pour la Population Française, pp. 30-36, Tec \& Doc, 3th edition, 2000.

[22] B. E. Ainsworth, W. L. Haskell, M. C. Whitt et al., "Compendium of physical activities: an update of activity codes and MET intensities," Medicine and Science in Sports and Exercise, vol. 32, no. 9, pp. S498-S504, 2000.

[23] Y. F. C. Chao, S. Y. Chen, C. Lan, and J. S. Lai, "The cardiorespiratory response and energy expenditure of Tai-ChiQui-Gong," American Journal of Chinese Medicine, vol. 30, no. 4, pp. 451-461, 2002.

[24] S. S. Hui, J. Woo, and T. Kwok, "Evaluation of energy expenditure and cardiovascular health effects from Tai Chi and walking exercise," Hong Kong Academy of Medicine, vol. 15, pp. $4-7,2009$.

[25] O. Wayne, "Assessment of physical function among older adults," in Mature stuff: Physical Activity for the Older Adult, D. K. Laslie, Ed., pp. 105-106, American Alliance for Health, Physical Education, Recreation and Dance, Reston, Va, USA, 1988.

[26] V. H. Heyward, Advanced Fitness Assessment Exercise Prescription, Burgess, 3rd edition, 1984.

[27] K. Häkkinen, M. Alen, M. Kallinen, R. U. Newton, and W. J. Kraemer, "Neuromuscular adaptation during prolonged strength training, detraining and re-strength-training in middle-aged and elderly people," European Journal of Applied Physiology, vol. 83, no. 1, pp. 51-62, 2000.

[28] R. Re, N. Pellegrini, A. Proteggente, A. Pannala, M. Yang, and C. Rice-Evans, "Antioxidant activity applying an improved ABTS radical cation decolorization assay," Free Radical Biology and Medicine, vol. 26, no. 9-10, pp. 1231-1237, 1999.

[29] K. Satoh, "Serum lipid peroxide in cerebrovascular disorders determined by a new colorimetric method," Clinica Chimica Acta, vol. 90, no. 1, pp. 37-43, 1978.

[30] A. E. Hak, K. H. Polderman, I. C. D. Westendorp et al., "Increased plasma homocysteine after menopause," Atherosclerosis, vol. 149, no. 1, pp. 163-168, 2000. 
[31] M. A. Forgione, A. Cap, R. Liao et al., "Heterozygous cellular glutathione peroxidase deficiency in the mouse: abnormalities in vascular and cardiac function and structure," Circulation, vol. 106, no. 9, pp. 1154-1158, 2002.

[32] S. Palazzetti, A. S. Rousseau, M. J. Richard, A. Favier, and I. Margaritis, "Antioxidant supplementation preserves antioxidant response in physical training and low antioxidant intake," British Journal of Nutrition, vol. 91, no. 1, pp. 91-100, 2004.

[33] L. Z. H. Zhou, A. P. Johnson, and T. A. Rando, "NFkB and AP-1 mediate transcriptional responses to oxidative stress in skeletal muscle cells," Free Radical Biology and Medicine, vol. 31, no. 11, pp. 1405-1416, 2001.

[34] H. Sharma, P. Datta, A. Singh et al., "Gene expression profiling in practitioners of Sudarshan Kriya," Journal of Psychosomatic Research, vol. 64, no. 2, pp. 213-218, 2008.

[35] G. R. Upchurch, G. N. Welche, A. J. Fabian et al., "Homocyst(e)ine decreases bioavailable nitric oxide by a mechanism involving glutathione peroxidase," Journal of Biological Chemistry, vol. 272, no. 27, pp. 17012-17017, 1997.

[36] N. Weiss, Y. Y. Zhang, S. Heydrick, C. Bierl, and J. Loscalzo, "Overexpression of cellular glutathione peroxidase rescues homocyst(e)ine-induced endothelial dysfunction," Proceedings of the National Academy of Sciences of the United States of America, vol. 98, no. 22, pp. 12503-12508, 2001.

[37] E. Lubos, J. Loscalzo, and D. E. Handy, "Homocysteine and glutathione peroxidase-1," Antioxidants and Redox Signaling, vol. 9, no. 11, pp. 1923-1940, 2007. 


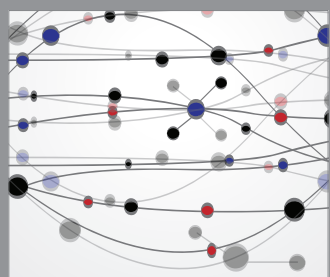

The Scientific World Journal
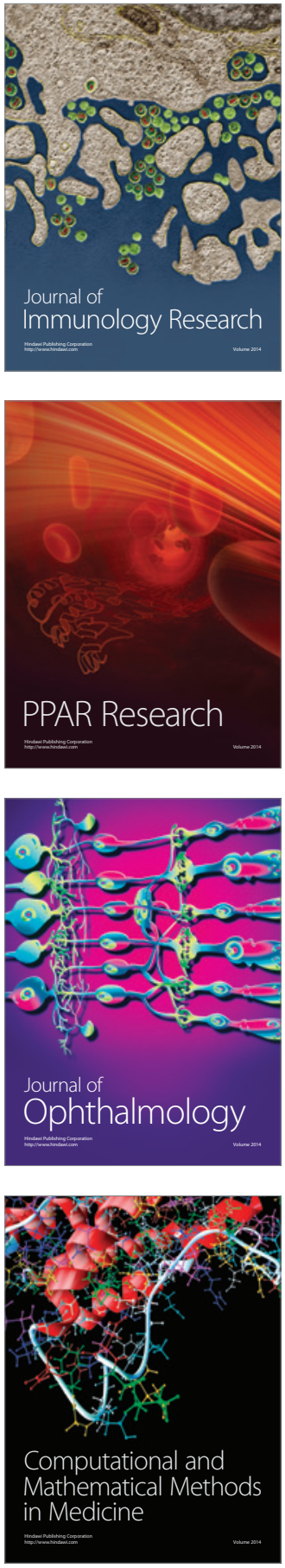

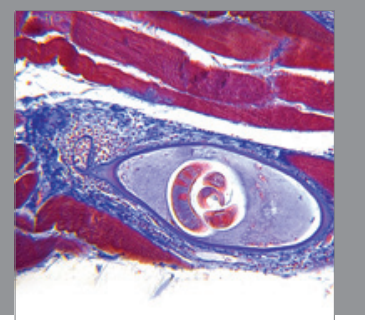

Gastroenterology

Research and Practice
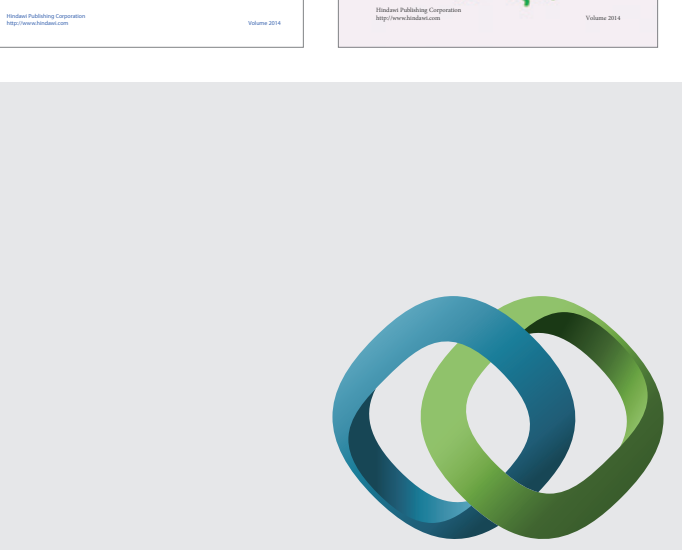

\section{Hindawi}

Submit your manuscripts at

http://www.hindawi.com
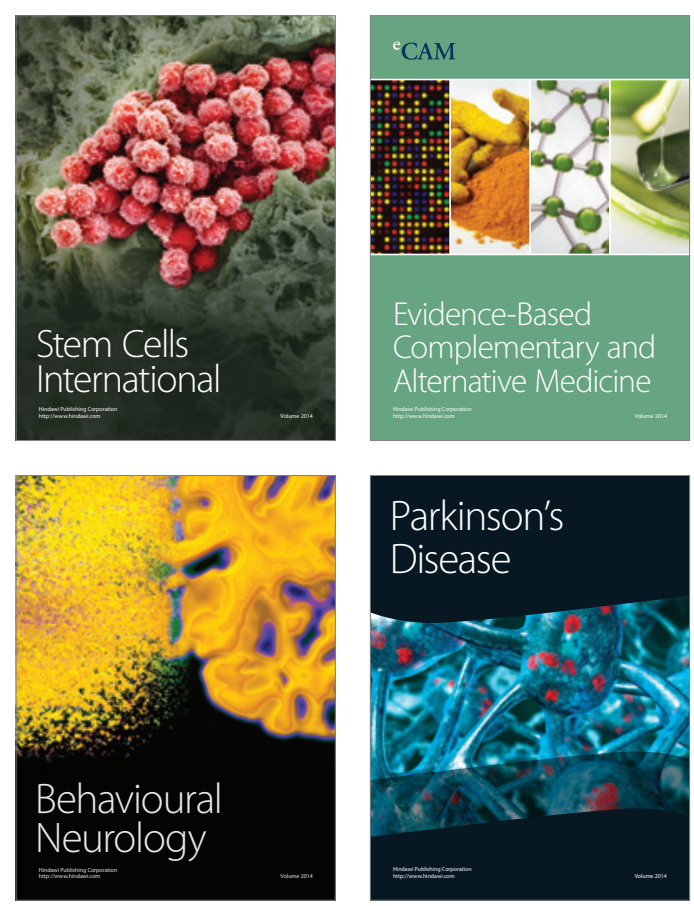

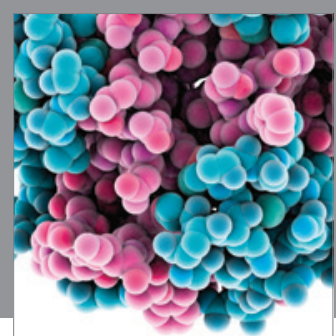

Journal of
Diabetes Research

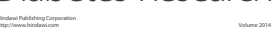

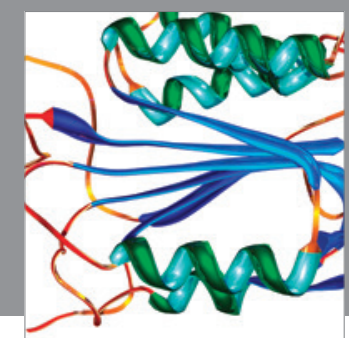

Disease Markers
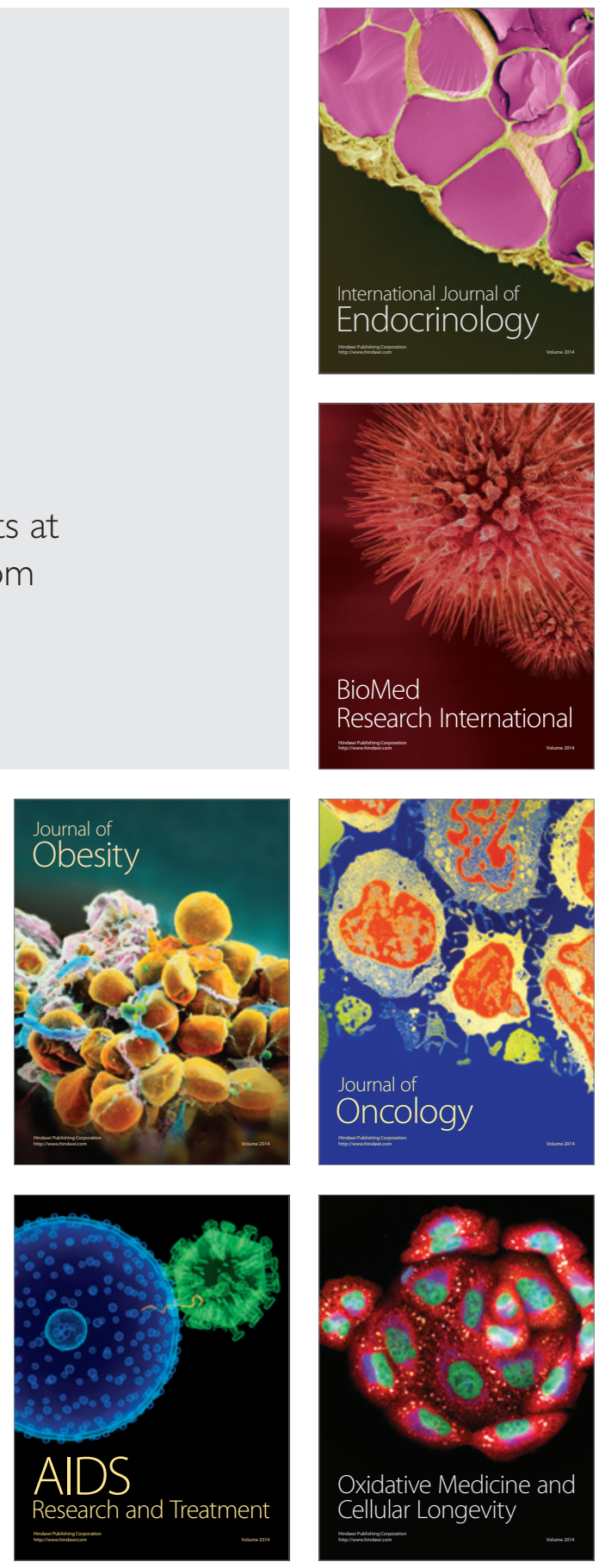